\title{
Italiens Regionen und deliberative Demokratie: Konzepte, Normenwerke, Anwendungsbeispiele
}

\section{Elisabeth Alber}

\section{Einleitung}

Vor dem Hintergund der Entwicklungen des italienischen asymmetrischen Regionalismus wird insbesondere in den letzten zwei Jahrzehnten wiederholt die Frage diskutiert, wie sich in Italiens Politik und Gesellschaft die Vorstellung von Demokratie und ihrer Ausgestaltung auf regionaler Ebene verändert hat. Neben der Föderalisierung hat die direkte Legitimation der politischen Leader durch die Wählerschaft an Bedeutung gewonnen und zur Entwicklung regionalpolitischer Autonomieverständnisse beigetragen. Deliberative Demokratieverständnisse in Form von Bürgerbeteiligungsverfahren sind dabei nur bedingt in Regionalstatuten oder regionalen, organischen Normenwerken verfasst. Sie bleiben in der Praxis meist ein Desiderat und sind nur in wenigen Regionen ein systemimmanenter Teil regionaler Politikgestaltung.

Dieser Beitrag geht auf das Konzept der deliberativen Demokratie und die Institutionalisierung deliberativer Bürgerbeteiligungsverfahren auf regionaler Ebene ein. Um die Relevanz des Konzepts und die bisher vereinzelten organischen Normenwerke im italienischen Regionalismus besser einordnen zu können, zeigen die Abschnitte 2 und 3 Grundsatzfragen zur Staatsstruktur Italiens und zur Rolle von Regionen auf. Laut derzeitigem Verfassungsgefüge sind letztere Dreh- und Angelpunkt im Regierungsund Verwaltungssystem, da sie über zahlreiche sowie Kernkompetenzen verfügen. Anschließend geht Abschnitt 4 auf die Frage ein, inwiefern ein deliberatives Demokratieverständnis Bestandteil von Regionalstatuten (Abschnitt 4.2) ist und wie regionale, organische Normenwerke (Abschnitte 4.3) aussehen. Eine kontextuelle Begriffsdeutung, das heißt eine Erläuterung der Frage, warum im laufenden italienischen Sprachgebrauch partizipative Demokratie auch deliberative Partizipationsverfahren umfasst, wird zuvor in Abschnitt 4.1 vorgenommen. Anschließend widmet sich Abschnitt 5 der Anwendung deliberativer Bürgerbeteiligungsverfahren zur Überarbeitung von Regionalstatuten. Insbesondere werden die Verfahren in den autonomen Provinzen Trento und Bozen analysiert (zwei Gebiets- 
körperschaften, die zusammen die Sonderstatutsregion Trentino-Südtirol bilden). Abschnitt 5 zeigt auf, dass Italien trotz seiner schwachen bzw. incomplete federality (hierzu Abschnitt 3) nicht nur im Bereich der regionalen, organischen Normenwerke zur Regelung deliberativer Partizipationsverfahren, sondern auch im Bereich substaatlicher Verfahren des participatory constitution-making eine interessante Fallstudie darstellt. Abschnitt 6 rundet den Beitrag mit einem Fazit zur Instiutionalisierung deliberativer Partizipationsverfahren ab.

\section{Der Verfassungsrahmen: Sonder- und Normalstatutsregionen}

Die italienische Verfassung (itVerf) unterscheidet zwischen 20 Regionen: den 15 Regionen mit Normalstatut ${ }^{1}$ stehen fünf Regionen mit Sonderstatut $^{2}$ gegenüber, die über eine weitaus größere Kompetenz- und Ressourcenausstattung verfügen, welche auch primäre, also ausschließliche Kompetenzen einschließt. Grundlegender Rechtsakt für jede der fünf autonomen Regionen ist das jeweilige Sonderstatut, welches Verfassungsrang besitzt und einerseits das Verhältnis der jeweiligen Region zum Staat (die Verteilung der Kompetenzen, die Finanzausstattung und die bilateralen Beziehungen), andererseits die interne Ordnung der jeweiligen autonomen Region regelt. Dies bedeutet zweierlei. Erstens, dass die Sonderstatuten der autonomen Regionen aus materieller Sicht die Grundgesetze der jeweiligen autonomen Gemeinschaft darstellen. Zweitens, dass die Sonderstatuten der autonomen Regionen aus formeller Sicht ein Rechtsakt des Staates sind, der nur mittels Verfassungsänderung gemäß Artikel 138 itVerf abgeändert werden kann - das heißt mittels einer Verabschiedung in doppelter Lesung in beiden Kammern des Parlaments in einem zeitlichen Abstand von mindestens drei Monaten. ${ }^{3}$ Dies ist ein Verfahren, das aufgrund der politischen Instabilität Italiens mühsam ist und auch Risiken

1 Die Regionen Piemont, Lombardei, Venetien, Ligurien, Emilia-Romagna, Toskana, Umbrien, Marken, Latium, Abruzzen, Molise, Kampanien, Apulien, Basilikata und Kalabrien verfügen über ein Normalstatut (statuto ordinario).

2 Friaul-Julisch Venetien, Sardinien, Sizilien, Trentino-Alto Adige/Südtirol und Aostatal/Vallée d'Aoste verfügen laut Art. 116 itVerf über besondere Formen und Arten der Autonomie gemäß Sonderstatuten. Die Region Trentino-Südtirol besteht aus den autonomen Provinzen Trient und Bozen (Art. 116 Abs. 2 itVerf), deren jeweilige umfassende Gesetzgebungs- und Verwaltungsautonomie unter dem gemeinsamen Dach der Region unterschiedlich ausdifferenziert ist.

3 Ein staatsweites Referendum, das bei Verfassungsänderungen verlangt werden kann, die mit lediglich einfacher Mehrheit in der zweiten Lesung verabschiedet 
für die Beibehaltung bzw. Erweiterung der Alleinstellungsmerkmale von Sonderstatutsregionen birgt. ${ }^{4}$

Eingerichet wurden die Sonderstatuten der autonomen Regionen Sizilien, Sardinien, Aostatal und Trentino-Südtirol mittels Abstimmung im Parlament im Zeitraum zwischen 1946-1948 (Friaul-Julisch Venetien im Jahr 1963). ${ }^{5}$ Die Statuten der anderen Regionen wurden hingegen erst im Jahr 1970 durch die damals erstmals gewählten Regionalparlamente selbst erarbeitet und nach der Überprüfung von Seiten des nationalen Gesetzgebers auf ihre Verfassungs- und Gesetzeskonformität per Regionalgesetz eingerichtet. ${ }^{6}$ Die fünf Sonderstatutsregionen, die im Sinn einer präventiven Strategie des Zentralstaates gegenüber separatistischen Tendenzen oder aufgrund des Vorhandenseins sprachlicher Minderheiten und internationaler Verpflichtungen eingerichtet worden waren, stellten folglich für lange Zeit die einzige Umsetzung der in der itVerf von 1948 festgeschriebenen Regionalautonomie dar. Sie verkörpern die erste Phase der Regionalisierung Italiens.

werden, ist bei einem statutsändernden Verfassungsgesetz ausgeschlossen (nicht zuletzt aufgrund des Minderheitenschutzes).

4 Dies trifft insbesondere für die Sonderstatutsregionen im Norden zu, welche ihre Handlungsspielräume, die aus den Statuten erwachsen, von Anfang an besser nutzten. Grundlegendes Instrument der Ausgestaltung kompetenzrechtlicher Handlungsspielräume von autonomen Regionen ist die Durchführungsbestimmung. Jede autonome Region verfügt über eine paritätisch von Vertretern des Staates und der Sonderstatutsregion besetzte Kommission, in der die Zuständigkeiten der jeweiligen autonomen Region näher definiert werden und die auch bei der Erweiterung einer Autonomie eine tragende Rolle spielt. Als atypische Rechtsquelle und aufgrund ihrer besonderen verfahrensrechtlichen Natur kann eine Durchführungsbestimmung, ausgearbeitet durch diese Kommission, nicht durch ein einfaches Staatsgesetz oder mittels eines Aktes mit Gesetzeskraft der Regierung abgeändert werden. Die autonome Provinz Bozen ist Spitzenreiter was die Anzahl von Durchführungsbestimmungen betrifft. Vgl. Francesco Palermo und Alice Valdesalici, »Irreversibly different. A country study of constitutional asymmetry in Italy«, in Patricia Popelier und Maja Sahadžić (Hrsg.), Constitutional Asymmetry in Multinational Federalism - Managing Multinationalism in Multi-tiered Systems, (Springer International Publishing, 2019), 287ff.

5 Die verfassungsgebende Versammlung verabschiedete die Sonderstatuten für die autonomen Regionen Sardinien (Verfassungsgesetz Nr.3/1948), Aostatal (Verfassungsgesetz Nr. 4/1948) und Trentino-Südtirol (Verfassungsgesetz Nr. 5/1948). Mit Verfassungsgesetz Nr.2/1948 wurde das 1946 mit gesetzesvertretendem Dekret Nr. 455/1946 verabschiedete Statut der Region Sizilien in ein Verfassungsgesetz umgewandelt. Die Errichtung der Region Friaul-Julisch Venetien wurde mit Verfassungsgesetz Nr. 1/1963 vollzogen.

6 Siehe Art. 123 itVerf für Details zum Verfahren. 
Die zweite Phase der Regionalisierung war gekennzeichnet durch den „Föderalismus per Dekret" ${ }^{\text {"7 }}$ - also der einfachgesetzlichen Übertragung von Aufgaben vom Staat auf die Regionen und örtlichen Körperschaften mittels der Verwaltungsreformen in den 1990er Jahren. ${ }^{8}$ In jenen Jahren blieb die nötige parallele Überführung der Beamtenschaft von den Zentralministerien an die Regionen als Träger der Kompetenzen jedoch aus. Ebenso folgten der Neufassung weitere Teile des V. Titels der itVerf durch die Verfassungsreform von 2001 - das heißt der Umkehr der Residualkompetenz, die seitdem den Regionen zuerkannt ist und somit dem Grundsatz einer Kompetenzverteilung zwischen dem Staat und den Regionen nach dem Subsidiaritätsprinzip gleichkommt ${ }^{9}$ - nur vereinzelt regionale Autonomieverständnisse in der Politik, auch wegen fehlender Finanzmittel bzw. der ausbleibenden Umsetzung des in der Verfassungsreform 2001 festgeschriebenen Finanzföderalismus ${ }^{10}$.

Seit der Verfassungsreform von 2001, die die dritte Phase der Regionalisierung Italiens eingeläutet hat, ermöglicht Art. 116 Abs. 3 itVerf auch allen Regionen mit Normalstatut, mit der Zentralregierung über „zusätzliche besondere Formen und Bedingungen der Autonomie“ zu verhan-

7 Vgl. Jens Woelk, »Föderalismus per Dekret? Zum Stand der Bassanini-Reformen«, (2000) 13 Jabrbuch für italienisches Recht $105 \mathrm{ff}$.

88 Die sogenannten Bassanini-Gesetze mit den entsprechenden Durchführungsverordnungen. Bezüglich der Regionen und Gemeinden siehe Gesetz Nr. 59/1997 und Gesetz Nr. 127/1997 sowie die Durchführungsverordnung Nr. 112/1998.

9 In diesen Neuerungen drückt sich die Auffassung einer Gleichstellung von Staat und Regionen aus, im Sinn funktionaler statt hierarchisch bestimmter Kompetenzbereiche. Hierzu trägt auch die Abschaffung der Präventivkontrolle regionaler Gesetze durch Zustimmung der staatlichen Regierung bei: Staat und Regionen sind im Hinblick auf die Gesetzgebung von nun an gleichgestellt. Eventuelle Ersetzungsbefugnisse des Staates, z.B. im Falle regionaler Untätigkeit bei der Umsetzung völkerrechtlicher oder europarechtlicher Verpflichtungen, stehen unter dem Vorbehalt ihrer Ausübung im Sinn des loyalen Zusammenwirkens, d.h. in Abstimmung mit den Regionen. Auch folgen Verwaltungszuständigkeiten nicht mehr den Gesetzgebungskompetenzen, sondern werden entsprechend der in Art. 118 genannten Kriterien (Subsidiarität, Differenzierung und VerhältnismäBigkeit) flexibel zugewiesen.

10 Gemäß Art. 119 itVerf verfügen die Gebietskörperschaften im Einklang mit der itVerf und nach einem staatlich vorgegebenen Koordinierungsschlüssel über Finanzautonomie in Bezug auf die Aufgaben in ihrem Zuständigkeitsgebiet. Die Neuregelung der Finanzbeziehungen der Gebietskörperschaften (Regionen, Provinzen, Großstädte mit Sonderstatus und Gemeinden) wurde ab 2009 mit der Verabschiedung des Rahmengesetzes Nr. 42/2009 und einer Reihe zu seiner Umsetzung notwendigen Regierungsdekreten angegangen. Infolge der Finanz- und Wirtschaftskrise geriet sie komplett ins Stocken. 
deln. ${ }^{11}$ In Bezug auf die Regionen mit Sonderstatut gilt weiterhin die Bestimmung laut Art. 10 des Verfassungsgesetzes Nr. 3/2001, die besagt, dass bis zur Anpassung der jeweiligen Statuten die Bestimmungen dieses Verfassungsgesetzes auch in den Regionen mit Sonderstatut und in den autonomen Provinzen Trient und Bozen Anwendung finden, und zwar für die Teile, in denen Formen der Autonomie vorgesehen sind, welche über die bereits zuerkannten hinausgehen. Die gemäß Art. 116 Abs. 3 itVerf erweiterte Autonomie für Regionen mit Normalstatut - bekannt unter dem Begriff des regionalismo differenziato (differenzierter Regionalismus) - ist eine zusätzliche Autonomieform neben jener der Regionen mit Sonderstatut. Sofern Regionen mit Normalstatut einen ausgeglichenen Haushalt vorweisen, können sie mittels eines Verhandlungsverfahrens im Bereich der konkurrierenden Gesetzgebungskompetenzen ${ }^{12}$ und für manche Sachgebiete auch im Bereich der ausschließlichen Gesetzgebungskompetenzen des Zentralstaates ${ }^{13}$ über mehr Zuständigkeiten und über besondere Arten der Autonomie verfügen.

11 Vgl. Carolin Zwilling, »Italiens Regionen zwischen Reform und Stillstand«, in Europäisches Zentrum für Föderalismus-Forschung Tübingen (Hrsg.), Jabrbuch des Föderalismus 2017. Föderalismus, Subsidiarität und Regionen in Europa (Nomos, 2017), 369ff.

12 Folgende Sachgebiete gehören zur konkurrierenden Gesetzgebung: die internationalen Beziehungen der Regionen und ihre Beziehungen zur Europäischen Union; Außenhandel; Arbeitsschutz und -sicherheit; Unterricht, unbeschadet der Autonomie der Schuleinrichtungen und unter Ausschluss der theoretischen und praktischen Berufsausbildung; Berufe; wissenschaftliche und technologische Forschung und Unterstützung der Innovation der Produktionszweige; Gesundheitsschutz; Ernährung; Sportgesetzgebung; Zivilschutz; Raumordnung; Häfen und Zivilflughäfen; große Verkehrs- und Schifffahrtsnetze; Regelung des Kommunikationswesens; Produktion, Transport und gesamtstaatliche Verteilung von Energie; Ergänzungs- und Zusatzvorsorge; Koordinierung der öffentlichen Finanzen und des Steuersystems; Aufwertung der Kultur- und Umweltgüter und Förderung und Organisation kultureller Tätigkeiten; Sparkassen; Landwirtschaftsbanken, Kreditinstitute regionalen Charakters; Körperschaften für Boden- und Agrarkredit regionalen Charakters. Unbeschadet der dem staatlichen Gesetzgeber vorbehaltenen Befugnis zur Festsetzung wesentlicher Grundsätze steht die Gesetzgebungsbefugnis für Sachgebiete der konkurrierenden Gesetzgebung den Regionen zu.

13 Im Bereich des Rechtsinstituts des Friedensrichters, im Bereich der allgemeinen Bestimmungen im Unterricht und in den Bereichen Umweltschutz, Ökosysteme und kulturelle Gemeingüter. 


\section{Der autonomiepolitische Rahmen: Italiens federality}

Von besonderem Gewicht für Aussagen über die föderale Kultur im italienischen Regionalismus sind Burgess' Überlegungen zur Bedeutung von politischer Kultur, das heißt von Einstellungsmustern und Deutungsnarrativen gegenüber den drei Dimensionen von Politik: ${ }^{14}$ Erstens polities als Institutionen und Strukturen (Form). Zweitens politics als akteursbezogene Politik (Prozess). Drittens policies als Politikgestaltung (Inhalt). Solche Einstellungsmuster und Deutungsnarrative fasst Burgess unter dem Begriff federality zusammen, der Aussagen darüber ermöglichen soll, wie föderal ein System ist. ${ }^{15}$

Im Konkreten bezieht Burgess' federality sich auf das Ausmaß, in dem politische Systeme föderale Werte und Praktiken wie gegenseitige Anerkennung, Dialog und Kompromissbereitschaft, aber auch Vertrauen und Reziprozität, aufrechterhalten und angewendet werden - Werte, die auch unerlässlich sind für jeden deliberativen Partizipationsprozess. In die Kategorie föderaler politischer Systeme gehören demnach laut Burgess nicht nur etablierte Föderalstaaten wie die USA oder auch Deutschland, sondern auch solche Staaten, die - wie Italien - im formellen Sinn keine Föderalstaaten sind, aber föderale Werte als Verfassungsprinzipien festgeschrieben haben und diese in der politischen Praxis leben - wenngleich in unzureichender Art und Weise als incomplete federal democracies. ${ }^{16}$

Italiens „föderaler Regionalismus"17 zeichnet sich bei der Errichtung der Normalstatutsregionen dadurch aus, dass das regionale, demokratiepolitische Gedankengut wie auch die sozio-kulturellen und geographischen Eigenschaften regionaler Gemeinschaften größtenteils nicht mit in die Statuten aufgenommen wurden. Aus diesem Grund sprach man Anfang der 1980er Jahre von „Regionen ohne Regionalismus“. ${ }^{18}$ Die Regionalisierung schritt zwar voran, doch entwickelten die Regionen mit Normalstatut nur vereinzelt ein autonomiepolitisches, regionales Bewusstsein. Gründe sind auch das Fehlen einer verfassungsrechtlich verankerten Regionenkammer auf zentralstaatlicher Ebene, das zentralistisch-angelegte Parteiensystem, welches nur bedingt Territorialinteressen kohärent fasst und vor-

14 Vgl. Michael Burgess, In Search of the Federal Spirit (OUP, 2012).

15 Ibid., 260ff.

16 Ibid., 273.

17 Günther Pallaver und Marco Brunazzo »Italy: The Pendulum of "Federal” Regionalism«, in Ferdinand Karlhofer und Günther Pallaver (Hrsg.), Federal PowerSharing in Europe (Nomos, 2017), 149ff.

18 Giorgio Pastori, »Le regioni senza regionalismo«, (1980) 2 ilMulino $204 \mathrm{ff}$. 
antreibt, und die faktisch komplex-verwobenen und politisch-bedingten Beziehungsgeflechte zwischen den Regionen einerseits, und zwischen der regionalen und der zentralstaatlichen Ebene andererseits. ${ }^{19}$

Die Entwicklung beider Kategorien von Regionen - Sonder- und Normalstatutsregionen - erfolgte daher nach der Verfassungsreform 2001 insbesondere durch die Verfassungsrechtsprechung und ist - aus politischer Sicht - an die Verhandlungsstärke regionaler Eliten gebunden. Zwar entwickelte sich trotz fehlender, klarer Vorgaben im Bereich der intergouvernementalen Beziehungen durch die conferenza stato-regioni-province autonome (Ständige Staat-Regionen-Autonome Provinzen Konferenz) ${ }^{20}$ und mit Bezug auf das verfassungsrechtlich verankerte Prinzip der loyalen Zusammenarbeit (Art. 120 itVerf) ein gewisser regionalismo cooperativo (kooperativer Regionalismus), doch fehlt weiterhin eine klare Gesamtstrategie im Sinn der federality von Burgess. Starke Asymmetrien und insbesondere die politische Instabilität wirken seit jeher auf Föderalisierungsreformen, intergouvernementale Beziehungen und die Entwicklung, aber auch gegenseitige Anerkennung, regionaler Autonomieverständnisse ein. ${ }^{21}$

Vor diesem Hintergrund hat die deliberative Demokratie als breit gefasstes Konzept zwar Eingang gefunden in die Regionalstatuten, doch sind deliberative Verfahren nur vereinzelt ein systemimmanenter Teil regionaler Autonomieverständnisse geworden. Italiens incomplete federal democracy hat nichtsdestotrotz wesentlich dazu beigetragen, dass einige Regionen im Bereich der Institutionalisierung deliberativer Bürgerbeteiligungsverfahren eine Vorreiterrolle einnehmen konnten - eben weil Regionen über weitreichende Handlungsspielräume verfügen und so ihre Politikgestaltung

19 Regionenpräsidenten können territoriale Interessen in Anbetracht des Fehlens einer Regionenkammer auf zentralstaatlicher Ebene nur bedingt in den Vordergrund rücken. Italiens Parlamentarismus ist gekennzeichnet durch den „perfekten Bikameralismus“, in dem die Abgeordnetenkammer und der Senat über dieselben Funktionen verfügen (mit dem Senat, der auf regionaler Basis gewählt wird). Zur Territorialisierung von Parteiagenden siehe Linda Basile, The Party Politics of Decentralization (Palgrave Macmillan, 2019).

20 Zur Rolle der Konferenz als schwaches, aber derzeit einziges Koordinierungsorgan siehe Carolin Zwilling und Elisabeth Alber, »Italien auf der Suche nach (s)einer föderalen Kultur: Konfrontation plus Konfliktregelung gleich Kooperation?« in Europäisches Zentrum für Föderalismus-Forschung Tübingen (Hrsg.), Jahrbuch des Föderalismus 2013. Föderalismus, Subsidiarität und Regionen in Europa (Nomos, 2013), 317ff.

21 Vgl. für viele Gianfranco Baldini und Brunetta Baldi, »Decentralization in Italy and the Troubles of Federalization", (2014) 1/24 Regional and Federal Studies $87 \mathrm{ff}$. Lutz Bergner, Der italienische Regionalismus (Verlag Dr. Kovač, 2008). 
mittels der Einbindung von Bürgern auch deliberativ-demokratisch angehen können. Die in Abschnitt 4.3 angeführten Beispiele bekräftigen dies, und sie bekräftigen ebenso die folgenden drei damit zusammenhängenden Thesen, die vermehrt in der weltweiten Literatur zu Föderalismus und (deliberativer) Demokratie diskutiert werden. Erstens, dass eine bundesstaatliche bzw. stark dezentralisierte Ausgestaltung eines Staates förderlich ist für die Institutionalisierung deliberativer Partizipationsverfahren: „federalism should be seen as, and can become, a method by which deliberative settings can be created because federalism localizes decision-making“. ${ }^{22}$ Zweitens, dass die Umsetzung von deliberativen Bürgerbeteiligungsverfahren der Wiederherstellung eines ungetrübten Verhältnisses zwischen Institutionen und Bürgern im Sinn eines „aktivierenden (Wohlfahrts)Staates" dient, ${ }^{23}$ das heißt einer neuen Verantwortungsteilung zwischen dem Staat und der Gesellschaft. Diese neue Verantwortungsteilung fußt auf dem Prinzip der Selbstregulierung, welches gesellschaftspolitische Akteure mittels vermehrter Aufgabenübernahme in die Problemlösung mit einbindet und die daraus resultierende Kollaboration einer staatlichen oder hierarchischen Steuerung vorzieht - nicht zuletzt aufgrund der Tatsache, dass kollaborative Entscheidungsfindungsprozesse Sozialkapital freischalten und somit Innovation generieren können. ${ }^{24}$ Drittens, dass eine bundesstaatliche bzw. stark dezentralisierte Ausgestaltung eines Staates Voraussetzung ist für bürgerschaftliches Engagement und bürgerschaftliche Kompetenz, zwei Elemente, die unerlässlich sind für die Umsetzung erfolgreicher Bürgerbeteiligungsverfahren. ${ }^{25}$ Das Konzept der bürgerschaftlichen Kom-

22 Kyle Scott, Federalism. A Normative Theory and its Practical Relevance, (Bloomsburg Publishing, 2011), 39. Vgl. auch Karl-Peter Sommermann »Citizen Participation in Multi-Level Democracies - An introduction«, in Cristina Fraenkel-Haeberle, Sabine Kropp, Francesco Palermo und Karl-Peter Sommermann (Hrsg.), Citizen Participation in Multi-Level Democracies (Brill-Nijhoff, 2015), $1 \mathrm{ff}$.

23 Bernhard Blanke und Stephan v. Bandemer, »Der „aktivierende Staat“", (1999) 6 Gewerkschaftliche Monatshefte 321ff. Irene Dingeldey, "Aktivierender Wohlfahrtsstaat und sozialpolitische Steuerung «, (2006) 8-9 Aus Politik und Zeitgeschichte $3 \mathrm{ff}$.

24 Arthur Benz spricht von innovationsgenerierendem Wettbewerb und vom Potential des institutional engineering zwischen allen Akteuren eines Staates, von kooperativen und deliberativen Entscheidungsfindungsprozessen; Arthur Benz, »Föderalismus und Demokratie - Eine Untersuchung zum Zusammenwirken zweier Verfassungsprinzipien«, (2003) 57 polis. Vgl. auch Arthur Benz und Nicolai Dose (Hrsg.), Governance - Regieren in komplexen Regelsystemen (VS Verlag für Sozialwissenschaften, 2010).

25 Vgl. Caroline Patsias, Anne Latendresse und Laurence Bherer, »Participatory Democracy, Decentralization and Local Governance«, (2013) 37(6) International Journal of Urban and Regional Research $2214 \mathrm{ff}$. 
petenz - civic competence - wird dabei als bindendes Element zwischen den zwei Verfassungsprinzipien der Demokratie und des Föderalismus angesehen und aus anwendungsorientierter Sicht als „institutional capacity of citizens to be actively engaged in the actual process of government" definiert. ${ }^{26}$

\section{Deliberative Demokratie auf regionaler Ebene}

Zur Klärung der Frage, inwiefern deliberative Demokratie ein Bestandteil regionaler Autonomieverständnisse ist, bedarf es dreierlei. Erstens, einer kontextuellen Begriffsdeutung mit Bezug auf anwendungsrelevante Spezifika. Zweitens, der Kontextualisierung deliberativer Demokratie im staatsrechtlichen und regionalstatutarischen Demokratieverständnis. Drittens, der Analyse regionaler, organischer Normenwerke zur Regelung deliberativer Bürgerbeteiligungsverfahren.

\subsection{Die Begriffsdeutung zu democrazia partecipativa}

In Bezug auf den italienischsprachigen Raum und italienischsprachiger Literatur zu deliberativen Bürgerbeteiligungsverfahren muss hinsichtlich der Begriffsdeutung festgehalten werden, dass für die Ausgestaltung deliberativer Demokratie meist der Begriff der partizipativen Demokratie verwendet wird, wobei mit democrazia partecipativa eben auch all jene Beteiligungsformen gemeint sind, die auf Deliberation fußen, also auf - bezugnehmend auf deutschsprachige Literatur - der „argumentative[n] Suche nach und Gewichtung von Gründen für und gegen Handlungsoptionen durch

26 Vgl. Kostas A. Lavdas und Dimitris N. Chryssochoou, »A Republic of Europeans: Civic Unity in Polycultural Diversity «, in Léonce Bekemans Maria KarasinskaFendler, Marco Mascia, Antonio Papisca, Constantine A. Stepannou und Peter G. Xuerebet (Hrsg.), Intercultural Dialogue and Citizenship: Translating Values into Actions (Marsilio, 2007). Ähnlich argumentieren aus rechtswissenschaftlicher Sicht Margherita M. Procaccini, »Partecipazione e federalismo: lessico e strumenti di un metodo di governo", in Alessandra Valastro (Hrsg.), Le regole della democrazia partecipativa (Jovene Editore: 2010), 57ff; und Umberto Allegretti, »Participatory Democracy in Multi-Level States«, in Cristina Fraenkel-Haeberle, Sabine Kropp, Francesco Palermo und Karl-Peter Sommermann (Hrsg.), Citizen Participation in Multi-Level Democracies (Brill-Nijhoff, 2015), $207 \mathrm{ff}$. 
eine Gruppe“ ${ }^{27}$ Die dialogische Deliberation bei solchen Beteiligungsprozessen, das heißt der kommunikative Austausch inhaltlicher Positionen, soll dabei der Logik der Öffentlichkeit folgen: „Die genannten Gründe verlangen nach Verallgemeiner- und prinzipieller Übertragbarkeit [wobei das offene Darlegen von Gründen der Prüfung von Verallgemeinerbarkeit und Übertragbarkeit dient]. Was für einen Teilnehmer ein Grund ist, muss für die anderen zumindest ein möglicher Grund sein. Private Motive und Interessen (z.B. der Wunsch nach mehr Geld oder Macht) gelten in der Deliberation nicht als legitime Gründe. " 28 Solch ein Austausch inhaltlicher Positionen ist gekennzeichnet durch zwei Qualitäten: eine intrinsische, die sich auf die subjektive Erfahrung der Teilnehmenden bezieht und dem zivilgesellschaftlichen Selbstverständnis des aktiven Bürgers Rechnung trägt, und eine instrumentelle, die sich auf die Vermutung bezieht, dass mittels Deliberation Ergebnisse erzielt werden, die höhere epistemische Qualität aufweisen und so auch höhere Legitimität für sich beanspruchen können. ${ }^{29}$

Die Verwendung des Begriffs democrazia partecipativa im italienischen Raum ist bedingt durch die Tatsache, dass im Italienischen das Verb deliberare im laufenden Sprachgebrauch gleichgesetzt ist mit „etwas beschließen, einen Beschluss fassen“ und nicht mit „beratschlagen, abwägen“ (wie im Deutschen, aber auch im Englischen, den zwei Sprachen, in denen der Begriff auf die klassische etymologische Wurzel des Wortes Deliberation verweist, welche mit dem Begriff das Abwägen von Vor- und Nachteilen zu einer Sachlage meint). Bobbio und Pomatto sprechen bei deliberativen Dialogmodellen daher von einem modello a confronto, das unterschiedliche Meinungen und Argumentationsstränge gegenüberstellt und in dem die Letztentscheidungsinstanz zunächst eine neutrale Rolle einnimmt. ${ }^{30} \mathrm{Sie}$ grenzen dieses Dialogmodell ab vom modello a pressione, welches sie als einen Oberbegriff ansehen für partizipative Beteiligungsformen, die Druck ausüben und nicht gekennzeichnet sind durch einen argumentativen Mei-

27 Claudia Landwehr, "Demokratische Legitimation durch rationale Kommunikation«, in Oliver W. Lembcke, Claudia Ritzi und Gary S. Schaal (Hrsg.), Zeitgenössische Demokratietheorie, Band 1: Normative Demokratietheorien (Verlag für Sozialwissenschaften, 2012), 360.

28 Ibid, 360.

29 Gary S. Schaal und Felix Heidenreich, Einführung in die Politischen Theorien der Moderne, 3. Auflage (Verlag Barbara Budrich, 2016), 266.

30 Luigi Bobbio und Gianfranco Pomatto, »Modelli di coinvolgimento dei cittadini nelle scelte pubbliche«, in Sergio Fabbrini (Hrsg.), Rapporto sulla qualità della democrazia in Trentino, (Provincia Autonoma di Trento, 2008), 29. 
nungs- und Willensbildungsprozess, der einer Präferenzgenese aber auch Präferenzveränderung gleichkommt. Letztere Beteiligungsformen - also Verfahren, die Druck ausüben - gehören zwar wie jene, die auf dialogische Deliberation hinauslaufen, einem sachlichen Demokratieverständnis an, ${ }^{31}$ doch sind sie den partizipativen Demokratietheorien der 1970er Jahre zuzuschreiben. Ihre Ausgestaltung äußert sich beispielsweise in Demonstrationen oder Tätigkeiten zivilen Ungehorsams, nicht unbedingt in Prozessen der Beratschlagung, in denen Argumente abgewogen werden bzw. "gut beratschlagt“ wird hinsichtlich einer noch zu treffenden Entscheidung in Politik oder Verwaltung.

Was man unter "guter Deliberation" versteht bzw. wie sich diese ausgestalten lässt, hängt vom Kontext und der Zielsetzung des jeweiligen deliberativen Bürgerbeteiligungsverfahren ab. Vergne betont, dass „gute Deliberationsprozesse" durch folgende fünf Elemente gekennzeichnet sind:

1) eine ausreichende Menge an Informationsvermittlung und -austausch zur Sachlage, zum Verfahren und zur Akteurskonstellation; 2) eine Phase der Debatte, die der Vorstellung von Meinungen und Argumenten dient (und somit der agonistischen Politik gleichkommt); 3) eine Phase des Dialogs, in der Argumente geprüft und nach Gemeinsamkeiten gesucht wird; 4) eine Phase der Priorisierung der erarbeiteten Empfehlungen; 5) eine Phase der Ergebnisproduktion, die drei Formen annehmen kann: die Erstellung eines Berichts (beispielsweise eines Bürgergutachtens), einer Entscheidungsgrundlage (beispielsweise eines Gesetzentwurfs) oder einer klärenden Entscheidung zu einer konkreten Sachlage mittels Konsens-, Kompromiss- oder Mehrheitsprinzip. ${ }^{32}$

Ein Streifzug durch einschlägige Werke in der italienischsprachigen Literatur zeigt auf, dass - wie anderswo - keine einhellige Meinung zu "guter Deliberation" vorherrscht. Es gilt folgendes: normative-theoretische Arbeiten umreißen "gute Deliberation" in der Regel als einen umfassenden und anspruchsvollen Begriff, während empirische Studien und insbesondere die politische Praxis gelegentlich fast jede Form der Kommunikation als Deliberation bezeichnen (z.B. auch Anhörungen). Aus diesem Grund argumentiert Floridia, dass deliberative Demokratie vieles sein kann, eben weil seine Vielschichtigkeit differenziert zur Reflektion und Anwendung kommt: „...deliberative democracy is $[. .$.$] a political idea that inspires a$

31 Im Gegensatz zum numerischen Demokratieverständnis, das sich auf den Wahlakt beschränkt.

32 Antoine Vergne, »Ausblick: Auf dem Weg zu erneuerten Qualitätsstandards für die Planungszelle«, in Hans-Liudger Dienel et al. (Hrsg.), Die Qualität von Bürgerbeteiligungsverfahren (oekom, 2014), 414. 
political and cultural movement for democratic reform; also a theoretical model and a paradigm for empirical research. Is this too much? Probably not, because, today, those who refer to deliberative democracy actually $d o$ at least one of these things." (Hervorhebung im Original). ${ }^{33} \mathrm{Um}$ der konzeptionellen Unbestimmtheit Einhalt zu gebieten, vertritt Floridia die These, sich nunmehr nicht mit der Frage der Essenz von Deliberation und deliberativer Demokratie auseinanderzusetzen, sondern mit dem Status, der ihr zugesprochen wird, und mit der Frage, wie sie ausgestaltet ist und auf ein Gesamtsystem einwirkt.

\subsection{Deliberative Demokratie in den Regionalstatuten und der Grundsatz des systemic approach}

Die Regionalstatuten „zweiter Generation“, die seit 2004 und damit nach dem deliberative turn in der Demokratietheorie verabschiedet wurden, 34 erwähnen letztere nicht expressis verbis. Andererseits verweisen sie alle neben den verschiedenen Instituten der direkten Demokratie - auf partizipative Demokratie als Kern des regionalen Autonomieverständnisses, das die Mitwirkung von Bürgern am öffentlichen Leben zum Ziel hat. ${ }^{35}$ Die Regionalstatuten verankern das Recht auf Mitwirkung sowohl allgemein oft wird ihm ein ganzer Artikel gewidmet - als auch speziell im Bereich von Verwaltungsverfahren oder hinsichtlich der Gesetzgebungstätigkeit. Dieses Recht auf Mitwirkung reduziert sich in der Praxis jedoch meist

33 Antonio Floridia, From Participation to Deliberation (ECPR Press, 2017), 308.

34 Um die Jahrtausendwende mehren sich die Auffassungen, dass die Ausgestaltung der Demokratie im Idealfall as deliberative as possible zu erfolgen habe und die deliberative Demokratietheorie tritt definitiv das Erbe der partizipativen Demokratietheorie an. Die Jahre des deliberative turn in der Demokratietheorie gehen einher mit der vermehrten Anwendung deliberativer Verfahren in der politischen Praxis. Auf verschiedenen Regierungsebenen werden deliberative Bürgerbeteiligungsverfahren zunehmend als komplementäre Elemente im Vorfeld politischer Entscheidungsfindung eingesetzt. Was man unter Deliberation im Detail versteht wird zwar differenzierter, aber weiterhin unterschiedlich ausgelegt. Vgl. für viele John Gastil und Peter Levine, The deliberative democracy handbook: strategies for effective civic engagement in the twenty-first century, (Jossey-Bass, 2005); Gregorio Arena, Cittadini Attivi (Editore Laterza, 2006); Luigi Bobbio, Amministrare con $i$ cittadini. Viaggio tra le pratiche di partecipazione in Italia (Rubettino, 2007); Norbert Kersting (Hrsg.), Politische Beteiligung. Einführung in dialogorientierte Instrumente politischer und gesellschaftlicher Partizipation (VS Springer, 2008).

35 Vgl. Patrizia Vipiana, "La democrazia deliberativa a livello regionale«, (2018) fasc 01, 28 febbraio, AIC-Osservtorio regionale. 
auf die Einbindung von Bürgern zur Klärung einer Sachlage in Form von Informationsveranstaltungen oder Anhörungen, wobei in erster Linie jene Personen(gruppen) angesprochen werden, die von der Sachlage unmittelbar betroffen sind. Es handelt sich also nicht um deliberative Bürgerbeteiligungsverfahren im engeren Sinn, also jene Beteiligungsformate, die sich am Ideal der "guten Deliberation“ in der Form von Meinungs- und Willensbildung bzw. Präferenzgenese und -veränderung orientieren.

Bisher werden solchen deliberativen Bürgerbeteiligungsverfahren im italienischen staatsrechtlichen Demokratieverständis im Vergleich zu Ausgestaltungsformen der repräsentativen (und direkten) Demokratie ein geringerer Stellenwert eingeräumt. Wenngleich deliberative Partizipationsverfahren in der Wissenschaft als Inbegriff eines „methodological exercise of revising the terms of shared rule and self-rule, i.e. to innovate multi-level decision-making processes in the interest of a better management of the res publica" 36 gedeutet werden, und man auch anerkannt hat, dass die Disziplinen der deliberativen Demokratietheorie und der empirischen Deliberationsforschung quer zu den etablierten Grenzziehungen der Sozialwissenschaften liegen müssen, ist die Institutionalisierung deliberativer Bürgerbeteiligungsverfahren noch ein junges, vereinzeltes Phänomen. ${ }^{37}$ Nicht zuletzt weil es ein schwieriges Unterfangen ist, sich dem Ideal der „guten Deliberation" anzunähern. Sich deswegen von einem (anspruchsvollen) deliberativen Demokratiekonzept zu verabschieden, wäre jedoch unangemessen. Denn es kann weder eine Demokratie ohne deliberative Prozesse geben, noch kann Demokratie in modernen, pluralen Gesellschaften allein auf „guter Deliberation“ beruhen. Da alle Verfahren der Entscheidungsfindung Defizite aufweisen, sollte jedes politische System ein „adäquates Mischungsverhältnis" von Verfahrensmodi andenken, um durch deren Kombination die bestmögliche Demokratiebilanz zu erzielen. ${ }^{38}$ Zudem muss

36 Elisabeth Alber und Alice Valdesalici, »Framing Subnational 'Institutional Innovation' and 'Participatory Democracy' in Italy: some Findings on Current Structures, Procedures, and Dynamics«, in Francesco Palermo und Elisabeth Alber (Hrsg.), Federalism As Decision-Making: Changes in Structures, Procedures and Policies (Brill-Nijhoff, 2015), 451.

37 Vgl. Alessandra Valastro, Le regole della democrazia partecipativa. Itinerari per la costruzione di un metodo di governo (Jovene, 2010). Cristina Fraenkel-Haeberle, »Experimente der Bürgerbeteiligung in Italien«, (2013) 2 der moderne staat - dms: Zeitschrift für Public Policy, Recht und Management 507ff.

38 Thorsten Hüller, »Deliberation oder Demokratie? - Zur egalitären Kritik an deliberativen Demokratiekonzeoten«, (2012) 2 Zeitschrift für Politische Theorie, 145f. Ähnlich argumentiert Frank Hendriks, Vital Democracy: A Theory of Democracy in Action (OUP, 2010). 
unterschieden werden, ob es sich bei der Herausforderung der Umsetzung deliberativer Verfahren um ein inhärentes Problem der Deliberation handelt oder um ein Problem, das der Kontextsensibilität deliberativer Verfahren zuzuschreiben ist - also der Umsetzung durch die Politik, aber auch durch die Gesellschaft. Hinterfragen sollte man deshalb nicht per se den Nutzen von Deliberation und deliberativen Bürgerbeteiligungsverfahren, sondern vielmehr die kontextuell vorgefertigten Meinungsinterdependenzen von Akteuren und Teilnehmenden. Die Analyse deliberativer Partizipationsverfahren muss daher ein besonderes Augenmerk auf kontextuelle Aspekte und auf Interdependenzen der sozio-politischen Wirklichkeit legen, die auf Beteiligungsverfahren einwirken. Der Grundsatz des systemic approach, so Floridia, räumt nicht nur die Möglichkeit ein, multiple Deliberationsarenen und deren Wechselwirkung während der einzelnen Phasen eines konkreten Verfahrens mit zu berücksichtigen, sondern fordert die Deliberationsforschung auch auf, Strukturelemente und Dynamiken des gesamten politischen Systems in den Fokus der Analyse zu nehmen. ${ }^{39}$

Vor diesem Hintergrund, also dem deliberative turn in der Demokratietheorie und dem systemic approach in der empirischen Deliberationsforschung, urteilte das italienische Verfassungsgericht im Jahr 2002, dass die Umsetzung des Grundsatzes der Volkssouveränität (verankert in Art. 1 Abs. 2 itVerf) nicht auf die Ausgestaltungsform der repräsentativen Demokratie limitiert sein darf, sondern dass die Mitwirkungsformen der Bürger sich auf das gesamte „Verfassungsgerüst“ erstrecken müssen (Urteil 106/2002 Punkt 3), auch um Art. 3 Abs. 2 der itVerf Rechnung zu tragen. In Zusammenhang mit dem Grundsatz der substanziellen Gleichheit verweist dieser indirekt auf die Grundsätze der partizipativen Demokratie, i.e. die effektive Teilhabe aller arbeitenden Mitbürger an der politischen, wirtschaftlichen und sozialen Ordnung. Diese Bestimmung hätte eigentlich eine ausreichende Grundlage für partizipative Demokratieformen darstellen können, doch verkannte man aufgrund des Fehlens klarer Richtlinien bzgl. der Ausgestaltung von Partizipationsverfahren im Laufe der Jahrzehnte ihren verfassungsrechtlichen Status bzw. ihre Legitimität im System, so Teile der Lehre schon in den 1960er Jahren. ${ }^{40}$

Im Jahr 2004 legte das Verfassungsgericht hinsichtlich der Verfassungsmäßigkeit einiger Bestimmungen zu deliberativen Partizipationsverfahren

39 Antonio Floridia, La democrazia deliberativa: teorie, processi e sistemi (Carocci Editore, 2012), 33.

40 Lelio Basso, »Per uno sviluppo democratico nell'ordinamento costituzionale italiano«, in AA.VV., Studi per il ventesimo anniversario dell'Assemblea Costituente, II Le libertà civili e politiche (Vallecchi, 1969), 15. 
im Regionalstatut von Emilia-Romagna ausdrücklich fest (Urteil Nr. 379/2004), dass die Institute der partizipativen Demokratie (i.e. öffentliche Debatte und andere Formen der Teilnahme bei der Umsetzung verfahrensrechtlicher Akte und gesetzgeberischer Maßnahmen) keineswegs darauf abzielen, die Tätigkeit der öffentlichen Verwaltung zu behindern oder zu verlangsamen, und dass diese ebensowenig das Ziel verfolgen, dem Regionalparlament seine gesetzgeberische Kompetenz zu entziehen. Vielmehr zielen sie darauf ab, das Beziehungsgeflecht zwischen regionalen Institutionen und den Bürgern zu verbessern und transparenter zu gestalten. Das Verfassungsgericht bestätigte somit den komplementären Charakter deliberativer Bürgerbeteiligungsverfahren und bekräftigte, dass die Regionen über Handlungsspielräume verfügen, um diese einzuführen und anzuwenden. Solche Handlungsspielräume sind nämlich Ausdruck mehrerer Verfassungsprinzipien: jener der Selbstverwaltung und Dezentralisierung, die in den Artikeln 5 und 114 der itVerf verankert sind, und jenem der horizontalen Subsidiarität (Art. 118 Abs. 4 der itVerf), wonach der Staat, die Regionen, die Großstädte und die Gemeinden das Ziel verfolgen, die autonome Initiative einzelner Bürger und Bürgergruppen zur Durchführung gemeinwohlorientierter Tätigkeiten zu fördern.

\subsection{Regionale, organische Normenwerke zur Regelung deliberativer Partizipationsverfahren}

Wie im vorherigen Abschnitt betont, verankern alle Regionalstatuten das Konzept der partizipativen Demokratie. Einige beziehen sich auf spezifische Verfahren. Beispiele hierfür sind die öffentliche Debatte im Regionalstatut der Emilia-Romagna (Art. 17), aber auch die öffentliche Debatte im Regionalstatut der Basilikata (Art. 16). Ob und in welchem Ausmaß die statutarische Verankerung des Rechts auf Mitwirkung in Regionalstatuten zur Verabschiedung eines organischen Normenwerks zu deliberativen Partizipationsverfahren geführt hat, zeigt dieser Abschnitt an einigen Beispielen auf. Als Fazit kann eingangs festgehalten werden, dass bisher wenige Regionen organische Normenwerke erlassen haben, die deliberative Bürgerbeteiligungsverfahren als breit angelegtes Instrument der regionalen Politikgestaltung und als einen fixen Bestandteil im regionalen Institutionengefüge vorsehen. ${ }^{41}$

41 Vgl. Marco Brunazzo, »Istituzionalizzare la partecipazione? Le leggi sulla partecipazione in Italia«, (2017) 3 Istituzioni del Federalismo 837ff. 
Hervorzuheben ist das Regionalgesetz Nr. 46/2013 für deliberative Verfahren der Toskana, das Deliberation als politisches Recht der Bürger institutionalisiert. ${ }^{42}$ Es ist ein organisches Normenwerk, das die Mitwirkung der Bürger mittels öffentlicher Debatten auf regionaler und lokaler Ebene vorsieht (potenziell hinsichtlich aller öffentlicher Entscheidungsfindungsprozesse). Es folgt dem Leitsatz der maximalen Inklusivität, der allen Bürgern, die für Arbeits- und Studienzwecke ihren Wohnsitz in der Toskana haben und all denjenigen, die von der zu entscheidenden Sachlage betroffen sind, eine gleichberechtigte Teilnahme am deliberativen Partizipationsverfahren garantiert. Diese maximale Inklusivität erfüllt zusätzlich den Zweck der gesellschaftlichen Integration und Kohäsion. Eine spezifische vom Regionalparlament eingesetzte und von der Politik unabhängige Regionalbehörde ist für die Umsetzung der deliberativen Beteiligungsprozesse zuständig. Sie prüft die eingereichten Projekte, erlässt Leitlinien zur Durchführung des Partizipationsverfahrens, bewertet das Ergebnis und legt dem Regionalparlament einen Jahresbericht vor. Konkret sieht das Regionalgesetz zwei Verfahren vor: die öffentliche Debatte bei großen Projekten mit erheblichen Auswirkungen bzw. großen Investitionssummen; und Partizipationsprozesse bei kleineren Projekten, die von einer Mindestanzahl von Bürgern, Lokalkörperschaften und von Schulen eingebracht werden können. Die Durchführung dieser letzteren Prozesse fußt auf einem Einvernehmensprotokoll zwischen den Akteuren und der Region. Das toskanische Regionalgesetz 2013 ist auch aus dem Grund interessant, weil ihm das „experimentelle“ Regionalgesetz Nr. 69/2007 vorausging, welches unter Einbeziehung der Ergebnisse eines zweijährigen deliberativen Bürgerbeteiligungsverfahren ausgearbeitet wurde und eine Auslaufklausel vorsah. ${ }^{43}$ Laut dieser Klausel wäre jenes Regionalgesetz Ende 2012 außerkraftgetreten, sofern das Regionalparlament gesetzgeberisch nicht erneut tätig geworden wäre.

Ein weiteres Beispiel für ein organisches Normenwerk zur Regelung deliberativer Partizipationsverfahren ist in der Region Emilia-Romagna zu finden. Regionalgesetz Nr. 15/2018 zielt auf die allumfassende Mitwirkung von (betroffenen) Bürgern an Entscheidungsfindungsprozessen ab, wobei potenziell alle regionalen Akte und Politiken Gegenstand sogenannter „organisierter Diskussionsrunden“ sein können. Der Leitsatz der maximalen

42 Vgl. Rodolfo Lewanski, »Institutionalizing Deliberative Democracy: The "Tuscany laboratory" ", (2013) 9(1) Journal of Public Deliberation.

$43 \mathrm{Vgl}$. Antonio Floridia, »La democrazia deliberativa, dalla teoria alle procedure. Il caso della legge regionale Toscana sulla partecipazione«, (2007) 5 Le Istituzioni del Federalismo $603 \mathrm{ff}$. 
Inklusivität gilt auch für dieses Normenwerk: alle Personen, die in irgendeiner Weise von einer Sachlage betroffen sind, sollen gleichberechtigt an Partizipationsverfahren beteiligt werden - nicht nur der Sachlage wegen, sondern zum Zwecke der Förderung einer aktiven Bürgerschaft. Beteiligungsverfahren können auf Initiative regionaler Institutionen, der Lokalkörperschaften und anderer öffentlicher oder privater Stellen eingeleitet werden. Unter gewissen Auflagen können Anträge für die Einleitung eines deliberativen Partizipationsverfahren auch von einzelnen Bürgern oder Bürgergruppen gestellt werden. Eine fachliche Behörde mit einem vom Regionalparlament ernannten Garanten für Partizipation als Vorsitzenden ist Dreh- und Angelpunkt für die Durchführung von Partizipationsverfahren. Das Regionalgesetz Nr. 15/2018 fußt auf denselben Prinzipien wie sein Vorgänger, das Regionalgesetz Nr.3/201044. Es sind dies: 1) die Verbesserung der demokratischen Entscheidungsqualität, 2) der Ausbau des sozialen Zusammenhalts und des gegenseitigen Vertrauens, 3) die Reduzierung der Dauer und Kosten von Entscheidungsprozessen durch die Erhöhung der Akzeptanz von Entscheidungen mittels zeitgerechter, strukturierter Einbindung diffuser Sachkompetenz. Im Jahr 2016 setzte man den Prozess zur Überarbeitung des Regionalgesetzes Nr. 3/2010 in Gang, auch weil ein solcher durch die im selben Regionalgesetz enthaltene Evaluierungsklausel vorgesehen war. In Anlehnung an die Erfahrung in der Toskana setzte man dem formellen Prozess zur Überarbeitung des Regionalgesetzes einen Ideensammlungsprozess voraus, der Bürgern und Interessensgruppen vorbehalten war.

Den Normenwerken aus der Toskana und der Emilia-Romagna ist, wie auch dem Normenwerk der Region Apulien (Regionalgesetz Nr. 28/2017), zusätzlich folgendes gemein: alle verwenden neben dem Begriff der partizipativen Demokratie (democrazia partecipativa) auch den Begriff der deliberativen Demokratie (democrazia deliberativa). Sie sprechen von innovativen Modellen der partizipativen und (!) deliberativen Demokratie. ${ }^{45}$ Ähnlich dem toskanischen Regionalgesetz verweist jenes in Apulien auch auf ein Recht auf Mitwirkung der Bürger. Zusätzlich spricht es aber von einer Pflicht der Mitwirkung für Bürger und Interessensgruppen an beteili-

44 Vgl. Marco Ciancaglini, »La democrazia partecipativa e concertazione. La legge regionale 3/2010 dell'Emilia Romagna«, (2011) 2 Istituzioni del Federalismo 215ff; Alessandro Mengozzi, »Idee democratiche e spazi politici della governance partecipativa«, (2011) 2 Istituzioni del Federalismo $255 \mathrm{ff}$.

45 Art. 13 Abs. 1 d) Regionalgesetz Emilia-Romagna Nr. 3/2010. Präambel des toskanischen Regionalgesetzes Nr.46/2013. Art. 1 Abs. 3 Regionalgesetz Apulien Nr. 28/2017. 
gungszentrierter Entscheidungsfindung im Zusammenhang mit dem Konzept der aktiven Bürgerschaft und der Nutzung von Gemeingütern. Des Weiteren ähneln sich das toskanische Normenwerk und jenes von Apulien hinsichtlich der obligatorischen Durchführung öffentlicher Debatten im Vorfeld von Großprojekten oder von Projekten, die in relevanter Art und Weise auf das Territorium und die Gesellschaft einwirken.

Vergleicht man die drei bisher erläuterten Normenwerke hinsichtlich der Verantwortlichkeiten bei der Organisation deliberativer Bürgerbeteiligungsprozesse ergibt sich folgendes Bild: in der Toskana verfügt die Regionalbehörde für Partizipation (ein kollegiales Organ, das aus Experten besteht und vom Regionalparlament für fünf Jahre eingesetzt wird) im Vergleich zum Garanten für Partizipation in der Emilia-Romagna (ein Beamter der regionalen Verwaltung im Zusammenspiel mit zwei vom Rat der Gemeinden designierten Beamten) und dem Büro für Partizipation in Apulien (bei der Regionalregierung angesiedelt) über den größten, von allen regionalen Akteuren unabhängigen Handlungsspielraum.

In der autonomen Provinz Bozen (Südtirol), Teil der autonomen Region Trentino-Südtirol, wurden Interessensvertreter auch zur Ideensammlung hinsichtlich der Erarbeitung eines Landesgesetzes zur Bürgerbeteiligung eingebunden. Ziel des vierstufigen Prozesses im Zeitraum 2014-2015 war es, „die Meinungen verschiedener Vertreter von Organisationen der Zivilgesellschaft zum Thema der direkten Demokratie im Spezifischen und der Bürgerbeteiligung im Allgemeinen zu hören und zu dokumentieren, und mit ihnen gemeinsam Vorschläge zum neuen Gesetz zu erarbeiten“. ${ }^{46}$ Zwar ist das Landesgesetz Nr. 22/2018 im weitesten Sinn eine Überarbeitung des Landesgesetzes Nr.11/2005, doch schrieb man mit ihm in zweierlei Hinsicht Demokratiegeschichte. Erstens ist die Ausarbeitung einer Landesgesetzvorlage mit einer breiten Anhörung der (organisierten) Zivilgesellschaft ein Novum für Südtirol. Zweitens sieht das Landesgesetz Nr.22/2018 erstmals ein deliberatives Bürgerbeteiligungsverfahren vor. Beide Demokratieformen, also Instrumente der direkten und der partizipativen Demokratie, sollen laut Art. 1 des Landesgesetzes in Ergänzung zur repräsentativen Demokratie als „Ausdruck des Bürgerwillens“ Teil des demokratischen Lebens im Lande sein und das Ziel verfolgen, „die Möglichkeiten und Ausdrucksformen demokratischer Entscheidungen in der auto-

46 Laut den Organisatoren kann die hohe Anzahl (mehr als 80 Personen) als deutliches Signal für das große Interesse an der Mitgestaltung politischer Entscheidungen gewertet werden. Blufink Sozialgenossenschaft, Hin zu einem neuen Gesetz zur direkten Demokratie, Bericht April 2015, 4. 
nomen Provinz Bozen zu erweitern, zu stärken und allgemein zugänglich zu machen“. Der Bürgerrat (Art. 2 Abs. 6) ist dabei als „ein moderiertes Beteiligungsverfahren anzuerkennen, bei dem Bürger ergebnisoffen über gemeinwohlrelevante Fragestellungen der Landes- und Gesellschaftsentwicklung beratschlagen“ und „gesellschaftliche Mitverantwortung“ übernehmen. Der Bürgerratsprozess soll dreistufig ablaufen (Art. 18 Abs. 1): die Durchführung eines Bürgerrats, ${ }^{47}$ das Abhalten einer öffentlichen Veranstaltung, die Übergabe der Ergebnisse an die Letztentscheidungsinstanz (über das Präsidium des Südtiroler Landtags) und die Veröffentlichung des Bürgerratsberichts im Internet auf der Webseite des Südtiroler Landtags. Als Vorbild dient der Bürgerratsprozess in Vorarlberg, der professionell begleitet wird und seit Längerem fixer Bestandteil des Vorarlberger Demokratieverständnisses ist. Die Planung und Durchführung des Bürgerrats in Südtirol soll durch das Büro für politische Bildung und Bürgerbeteiligung erfolgen. Dessen Zusammensetzung und Verantwortlichkeiten werden im konkordanzdemokratischen politischen System Südtirols, das auf der Institutionalisierung dreier Sprachgruppen fußt (siehe Näheres in Abschnitt 5) kontrovers diskutiert. Der Bürgerrat, der die Angelegenheiten der Landesgesetzgebung oder der Landesverwaltung betreffen kann, soll gemäß Art. 19 Abs. 1 auf Antrag von 300 Bürgern abgehalten werden und als Ergebnis eine einstimmige, gemeinsam verfasste Erklärung erzielen. Diese Zielsetzung, das heißt eine einstimmige, gemeinsame Erklärung, scheiterte im Autonomiekonvent, dem deliberativen Partizipationsverfahren, das 2016-2017 südtirolweit zur Erarbeitung von Ideen für die Überarbeitung des Sonderstatuts der Region Trentino-Südtirol durchgeführt wurde (siehe Abschnitt 5). Wenngleich die Empfehlungen des Bürgerrats rechtlich unverbindlich sind, so schreibt das Landesgesetz in Art. 23 Abs. 3 fest: „Beinhaltet der Bürgerratsbericht konkrete Anregungen zur Landesgesetzgebung oder zur Landesverwaltung im Bürgerratsbericht, werden diese vom Präsidium des Landtags bzw. von der Landesregierung innerhalb von 60 Tagen behandelt. Über das Ergebnis der Behandlung werden die Initiatoren des Bürgerrats, die Teilnehmer des Bürgerrats und die Öffentlichkeit in geeigneter Weise informiert."

47 Ein Bürgerrat soll aus einer Gruppe von mindestens 12 Personen bestehen, die in der Meldeliste Südtirols eingetragen sind und das 16. Lebensjahr angetreten haben. Die Auswahl dieser Personen soll nach einem geschichteten Zufallsverfahren erfolgen (unter Berücksichtigung der Sprachgruppe, des Geschlechts und des Alters). Die Teilnahme am Bürgerrat soll freiwillig, aber verbindlich sein, und sich auf einen Zeitraum von eineinhalb Tagen erstrecken. 
Die autonome Provinz Trento (Trentino), die zusammen mit der autonomen Provinz Bozen die autonome Region Trentino-Südtirol bildet, gilt als Beispiel für die Institutionalisierung deliberativer Partizipationsverfahren auf überörtlicher Ebene. ${ }^{48}$ Zwar hat der Landesgesetzgeber kein (!) organisches Normenwerk zu deliberativer Demokratie verabschiedet, doch beinhaltet die umfassende Reform der lokalen Verwaltung innovative Ansätze für deliberative Bürgerbeteiligungsformen, die dem systemic approach zuzuschreiben sind. Das Trentino steht nicht nur exemplarisch dafür, wie eine Reform lokaler Körperschaften zur Institutionalisierung deliberativer Partizipationsverfahren führen kann, sondern auch dafür, dass eine solche Reform Potential für innovative, partizipative Governanceansätze in (dünn besiedelten) Berggebieten hat. Die Trentiner Talgemeinschaften (comunità di valle) wurden mit Landesgesetz Nr. 3/2006 als eine behördliche Ebene zwischen der Gemeinde und der Landesebene eingerichtet. ${ }^{49}$ Ziel war es, einen Teil der Zuständigkeiten der autonomen Provinz Trento auf die Gemeinden (im überörtlichen Verbund) zu übertragen. Teile der Bestimmungen des Landesgesetzes Nr.3/2006 wurden durch das Landesgesetz Nr. 12/2014 abgeändert. Es handelt sich dabei um die Bestimmungen, die deliberative Partizipationsverfahren vorsehen. Im Absatz Capo V ter-partecipazione nelle comunità verweist das Landesgesetz nämlich auf Bestimmungen bezüglich der Teilnahme der Bürger an den Entscheidungsfindungsprozessen der Talgemeinschaften. (Artikel 17 decies - 17 sedecies). Partizipationsberechtigt an den Diskussionsarenen sind Personen ab dem 16. Lebensjahr und die für den jeweiligen Prozess relevanten Interessensgruppen. Die Beteiligungsverfahren können auf Initiative der Gemeinden, der Talgemeinschaften, der Interessensgruppen und von mindestens fünf Prozent der Einwohnerschaft einer Talgemeinschaft beantragt werden. Eine unabhängige Behörde für Partizipation, auf fünf Jahre von der Trentiner Landesregierung nominiert und bestehend aus einem Beamten aus der Landesverwaltung und zwei Experten im Bereich deliberativer Partizipati-

48 An dieser Stelle muss angemerkt werden, dass die autonomen Regionen - darunter auch Trentino-Südtirol - anders als die Regionen mit Normalstatut über die ausschließliche Gesetzgebungskompetenz im Bereich der Ordnung der örtlichen Körperschaften verfügen.

49 Vgl. Sara Parolari und Alice Valdesalici, „Le Comunità di Valle: prime prove di attuazione della riforma istituzionale dell'autonomia speciale trentina«, (2011) 2 Istituzioni del Federalismo $423 \mathrm{ff}$. 
onsverfahren, ist einerseits für die (obligatorischen) ${ }^{50}$ dialogischen Prozesse zuständig, andererseits kann sie Prozesse auch selbst initiieren. Die Ergebnisse der deliberativen Verfahren sind beratender Natur, doch muss die Nichtberücksichtigung der Ergebnisse (auch eine eventuelle Abweichung von den Ergebnissen) von der Letztentscheidungsinstanz schriftlich begründet werden.

\section{Deliberative Verfahren bei der Überarbeitung von Regionalstatuten am Beispiel des Südtiroler Autonomiekonvents und der Trentiner Consulta}

Die Einbindung von Bürgern in deliberative Partizipationsverfahren zur Überarbeitung von Regionalstatuten ist vereinzelt in einigen Regionen mit Sonderstatut erfolgt. Vom Verfahren her gehören diese Versuche den Konventsmodellen (das heißt Versammlungsmodellen) an, welche bei grundlegenden Reformen wie Verfassungsreformen eingesetzt werden. Die Lehre spricht vom constitutional turn bei deliberativen Bürgerbeteiligungsverfahren $^{51}$ und von participatory constitution-making/constitutional change. ${ }^{52}$ Fasst man constitutional turn und participatory constitution-making breit, so können unter ihnen alle deliberativen Bürgerbeteiligungsverfahren subsumiert werden, die im Vorfeld jener politischer Entscheidungen durchgeführt werden, die für das jeweilige politische System grundlegende Veränderungen mit sich bringen. Durch das partielle „outsourcing "53 von Revisionsverfahren soll ein gesellschaftspolitischer konstitutioneller Moment geschaffen werden, in welchem die Bürger über neue Grundregeln bzw. die zukünftige Ausrichtung bestehender Grundregeln wie dem Verhältnis der Bürger zu den Institutionen beratschlagen.

Die beiden Verfahren in der autonomen Region Trentino-Südtirol bestanden aus einem mehrstufigen Prozess mit, im Falle des Autonomiekonvents in Südtirol, einer vorgeschalteten Ideensammlungsphase durch

50 Für gewisse Sachbereiche wie die Genehmigung von Sozialplänen, Leitfäden zur wirtschaftlichen Entwicklung sowie bestimmten Investitionen auf dem Territorium sind die Beteiligungsverfahren verpflichtend.

51 Vgl. Min Reuchamps und Jane Suiter (Hrsg.), Constitutional Deliberative Democracy in Europe (ECPR Press, 2016), 1.

52 Vgl. Xenophon Contiades and Alkemene Fotiadou (Hrsg.): Participatory Constitutional Change (Routledge, 2017); Umberto Allegretti, »Recenti costituzioni partecipate in Islanda, Ecuador, Bolivia«, (2013) 3 Quaderni Costituzionali 689ff.

53 Francesco Palermo, »Verso una riforma partecipata dello Statuto di Autonomia. Una prospettiva metodologica e comparata«, (2015) 3 Informator. Rivista giuridico amministrativa per il Trentino-Alto Adige 12. 
Bürger und, im Falle der Consulta im Trentino, einer zwischengeschalteten Mitwirkungsphase von Bürgern. Die den Diskussionsgremien vorbzw. zwischengeschalteten mehrmonatigen Partizipationsphasen und, im Falle des Autonomiekonvents auch die direkte Einbindung der Bürger in allen Gremien des Autonomiekonvents, sind mitunter jene Merkmale, die den Autonomiekonvent und die Consulta von ähnlichen Verfahren deliberativer Bürgerbeteiligung zur Überarbeitung der Statuten in anderen Regionen unterscheiden. In anderen autonomen Regionen wurden Bürger bei Verfahren mit derselben Zielsetzung zwar in die Arbeiten eingebunden, aber sie nahmen weder als offizielle Mitglieder in den Beratungsgremien teil, noch waren sie in mehrmonatige, vorgeschaltete Meinungsund Willensbildungsphasen oder in strukturierte Informations- und Anhörungsveranstaltungen, deren Organisation sich über das gesamte regionale Gebiet erstreckte, eingebunden.54

Weitere Alleinstellungsmerkmale, die den Autonomiekonvent und die Consulta kennzeichnen, sind die Sonderstellung der autonomen Provinzen Trentino und Südtirol im territorialen Staatsaufbau und die Tatsache, dass die beiden Verfahren in einem Minderheitengebiet durchgeführt wurden. Dabei ist Südtirols Autonomieverständnis im Vergleich zu jenem im Trentino stark von konkordanzdemokratischen Anpassungen in den Institutionen geprägt. ${ }^{55}$

Südtirol und das Trentino sind zwei autonome Provinzen, die zusammen die autonome Region Trentino-Südtirol bilden (eine von fünf Sonderstatutsregionen). Das Autononomiestatut der Region, das heißt ihr „Grundgesetz", geht in seiner heutigen Fassung auf das Jahr 1972 zurück. Es ist das Ergebnis von Verhandlungen, die zwischen den italienischen und österreichischen Regierungen unter mittelbarer Beteiligung der Süd-

54 Mit Regionalgesetz Nr. 12 vom 2. April 2004 wurde die Einrichtung eines Konvents in Friaul-Julisch Venetien veranlasst. Der Konvent tagte 5 Monate und bestand aus 50 Mitgliedern. William Cisilino, »Die Versammlung zur Ausarbeitung des neuen Sonderstatuts der Region Friaul-Julisch Venetien«, in Thomas Benedikter (Hrsg.), Mit mehr Demokratie zu mehr Autonomie - Bürgerinnen und Bürger reden mit (Politis, 2014), 33ff. Zum Konvents-Versuch im Aostatal Roberto Louvin, »Die Modernisierung der Sonderstatuten zwischen Pragmatismus und Wunschdenken «, im selben Band, 39ff.

55 Vgl. Günther Pallaver, »Das politische System Südtirols: Komplexe Machtteilung und ethnische Einflusssphären«, in Hermann Atz, Max Haller und Günther Pallaver (Hrsg.), Ethnische Differenzierung und soziale Schichtung in der Südtiroler Gesellschaft. Ergebnisse eines empirischen Forschungsprojekts (Nomos, 2016), 61ff. Siehe auch Jens Woelk, Francesco Palermo und Joseph Marko (Hrsg.), Tolerance through Law. Self Governance and Group Rights in South Tyrol (Brill-Nijhoff, 2008). 
tiroler Volkspartei (SVP) und anderer regionalpolitischer Eliten geführt worden waren. ${ }^{56}$ Diese Verhandlungen führten 1969 zum Abschluss des „Pakets“, welches über 80 Änderungen am Autonomiestatut von 1948 und weitere Maßnahmen zu deren Umsetzung vorsah. Damit sollte die im Gruber-De Gasperi Abkommen von $1946^{57}$ auf internationaler Ebene versprochene Autonomie für deutschsprachige Südtiroler in Italien in einem zweiten Schritt umgesetzt werden. Das Autonomiestatut von 1948 und dessen Umsetzung war hinsichtlich der völkerrechtlich verankerten Rechte der deutschsprachigen Südtiroler - Gleichstellung der deutschen und der italienischen Sprache in den öffentlichen Ämtern, Recht auf muttersprachlichen Unterricht, Gleichberechtigung bei der Zulassung zu öffentlichen Ämtern zum Zwecke eines angemessenen Proporzes - unzureichend. ${ }^{58}$

Die vielfältigen Änderungen im Autonomiestatut $1972^{59}$ sehen für die autonome Provinz Bozen ein konkordanzdemokratisches System zur Regelung des Zusammenlebens der Sprachgruppen sowie den für die Umsetzung eines solchen Systems nötigen Übergang der Kompetenzen von der Region auf die beiden autonomen Provinzen vor. Trentino und Südtirol, wenngleich unter dem gemeinsamen Dach der Region, entwickelten dadurch unterschiedliche Systeme. Während Südtirols Autonomie durch die Institutionalisierung von drei Sprachgruppen (Deutsch-, Italienisch- und Ladinischsprachige) ${ }^{60}$ und deren (historischen) Beziehungen geprägt ist, hat das Trentino eine überwiegend italienischsprachige Bevölkerung und

56 Vgl. Elisabeth Alber, »South Tyrol's Negotiated Autonomy«, (2017) 78 Journal of Ethnic Studies 41ff.

57 Die Friedensverhandlungen im Frühjahr 1946 machten deutlich, dass es zu keiner Veränderung der Brennergrenze kommen würde. Südtirol, das nach dem ersten Weltkrieg von Italien annektiert wurde und zuvor mit dem Trentino zu Österreich-Ungarn gehörte, würde bei Italien bleiben. Die Alliierten drängten Österreich und Italien zu einem Abkommen, das der deutschsprachigen Minderheit eine weitreichende Autonomie zusichern sollte. Die Außenminister der beiden Länder, Karl Gruber und Alcide De Gasperi, unterzeichneten das Abkommen am 5. September 1946, welches als Annex im italienischen Friedensvertrag aufgenommen wurde.

58 Vgl. Georg Grote, The South Tyrol Question, 1866-2010: From National Rage to Regional State (Peter Lang, 2012).

59 Verfassungsgesetz D.P.R. vom 31. August 1972, Nr. 670.

60 Im Rahmen der Volkszählung 2011 ergab der Sprachgruppenproporz folgende Zusammensetzung: 69,41\% für die deutsche Sprachgruppe, 26,06\% für die italienische Sprachgruppe und 4,53\% für die ladinische Sprachgruppe. 
lediglich drei kleine Sprachgruppen (Ladiner, Bernstoler, Zimbern), die der Trentiner Landesgesetzgeber punktuell anerkennt und fördert. ${ }^{61}$

Was die Änderung des Sonderstatuts der Region Trentino-Südtirol angeht, müssen die politischen Eliten beider autonomer Provinzen von den zentralstaatlichen Institutionen mit eingebunden werden. Die autonomen Provinzen haben überdies auch ein Initiativrecht für Änderungen, welches sie durch das Regionalparlament (zusammengesetzt aus den 35 Abgeordneten des Südtiroler Landtags und den 35 Abgeordneten des Trentiner Landtags) ausüben können und dem einzelne Beratungen in den Landtagen der beiden autonomen Provinzen vorausgehen.

Die Arbeiten des Autonomiekonvents und der Consulta, ${ }^{62}$ die mit Landesgesetzen in Südtirol und dem Trentino unterschiedlich geregelt wurden, ${ }^{63}$ fanden außerhalb der parlamentarischen Arbeiten in den jeweiligen Landtagen statt und waren damit nicht Teil des formellen Verfahrens zur Änderung des Sonderstatuts der autonomen Region Trentino-Südtirol. Nennenswert ist, dass kein regionales Verfahren angestrebt wurde, die Verfahren aber faktisch im selben Zeitraum 2016-2018 durchgeführt wurden (vor dem Hintergrund eines zentralstaatlichen Verfassungsreformvorhabens). ${ }^{64}$ Durch die getrennten Verfahren blieb die Lösung des Koordina-

61 Vgl. Simone Penasa, »From Protection to Empowerment through Participation: The Case of Trentino - a Laboratory for Small Groups«, (2014) 13 (2) JEMIE $30 \mathrm{ff}$.

62 Details zu den Arbeiten und Ergebnissen sind auf den Archivseiten der Verfahren einsehbar. Autonomiekonvent: www.konvent.bz.it/de.html; Consulta: www.rifor mastatuto.tn.it Die Mitarbeit in allen Gremien war ehrenamtlich und freiwillig.

63 Die politische Ausgangslage für die Verabschiedung der Landesgesetze war sehr unterschiedlich. In Südtirol, seit jeher durch den Grundsatz der geteilten ethnischen Governance geprägt, war die Einsetzung des Autonomiekonvents bereits im Koalitionsabkommen für die Legislaturperiode 2013-2018 (Südtiroler Volkspartei, SVP, mit der demokratischen Partei, PD) als Ziel genannt worden. Das Landesgesetz Nr.3/2015 zur Einsetzung des Konvents wurde alleinig mit den Stimmen der Regierungskoalition verabschiedet. Im Trentino wurde Landesgesetz Nr. 1/2016 zur Einsetzung der Consulta hingegen ohne Gegenstimmen verabschiedet.

64 Art. 1 des Trentiner Landesgesetzes nimmt im Gegensatz zu jenem in Südtirol ausdrücklich auf das Verfassungsreformvorhaben Renzi-Boschi Bezug, welches unter anderem das Verhältnis von Staat und Regionen neu regeln wollte und für die Sonderstatutsregionen eine Verpflichtung zu - späterer - Anpassung ihrer Statuten vorsah. Vgl. Elisabeth Alber, »Regionalstaat Italien - Reformblockaden und Perspektiven«, (2018) 1 Gesellschaft • Wirtschaft • Politik (GWP) $75 \mathrm{ff.}$ Für eine Bilanz zu den Reformen und Reformversuchen der Regierung Renzi vgl. Alexander Grasse, Markus Grimm und Jan Labitzke (Hrsg.), Italien zwischen Krise und Aufbruch, (SpringerVS, 2018). Der Konvent der 33 und die Consulta verlängerten 
tionsproblems aus und die Einigung auf einen einzigen Vorschlag wurde der Politik auf Landes- und Regionalebene überlassen.

Die zuvor beschriebenen Alleinstellungsmerkmale der autonomen Provinzen Trentino und Südtirol - insbesondere Südtirols „Sonderstellung in der Sonderstellung" 65 - sind der Grund dafür, warum kein regionales Verfahren angestrebt wurde. Die Region als Regierungsebene wurde mit dem Autonomiestatut von 1972 schrittweise ausgehöhlt und ihrer Kompetenzen entkleidet. Die Frage nach der Daseinsberechtigung der Region als Klammer der beiden autonomen Provinzen wurde in den Gremien des Autonomiekonvents und in der Consulta auch unterschiedlich gewertet. Aus Trentiner Sicht ist die regionale Regierungsebene für die Entwicklung und Garantie der Trentiner Autonomie unverzichtbar. Die gemeinsame geschichtliche Vergangenheit des Grenzgebiets Südtirol-Trentino und die internationale Verankerung der Autonomie durch das Gruber-De Gasperi Abkommen sind für das Trentino die Grundlagen für eine weitere Stärkung der Autonomie. In den Gremien des Autonomiekonvents wurde die Rolle der Region mehrheitlich nicht in diesem Sinn disktutiert. Im Gegenteil: Aufgrund der Entwicklungen der beiden autonomen Provinzen könnte die Region als Klammer abgeschafft werden. ${ }^{66}$ Auch wurden die Reformvorschläge zur ladinischsprachigen Bevölkerung, deren Siedlungsgebiete beide Provinzen umfassen und auch darüber hinausgehen, im Autonomiekonvent mehrheitlich unter Ausklammerung der Region als potenziell interessantes Dach diskutiert. Unterschiede (in Argumentationssträngen) gab es auch beim Thema des Minderheitenschutzes: Während die Consulta umfangreiche Vorschläge zur Aufwertung der drei kleinen Sprachgruppen und im Allgemeinen zur Mehrsprachigkeit im Schulwesen und anderen Bereichen im Sinn einer pluralen Gesellschaftsordnung vorbrachte, bestanden in Südtirol die Gremien des Autonomiekonvents mehrheitlich auf der strikten Auslegung bzw. Beibehaltung der proporzmäßi-

ihre Arbeiten um einige Monate, da die vorgesehene Jahresfrist zu knapp bemessen war.

65 In Anlehnung an Roberto Toniatti, der die autonnome Provinz Bozen als „Besonderheit in der Besonderheit" bezeichnet. Roberto Toniatti, »Un nuovo intervento della Corte in tema di rappresentanza politica preferenziale delle minoranze linguistiche: il consolidamento della democrazia consociativa etnica nel Trentino-Alto Adige«, (1999) 2 Le Regioni 291.

66 Vgl. Sara Parolari, »Il futuro dell'ente regionale come chiave di volta della riforma dello Statuto del Trentino-Alto Adige/Südtirol«, in Elisabeth Alber, Alice Engl und Günther Pallaver (Hrsg.), Politika 2018. Südtiroler Jabrbuch für Politik, (Raetia, 2018), $179 \mathrm{ff}$. 
gen oder paritätisch ausgestalteten Schutzmechanismen (unter anderem dem getrenntem Schulsystem). ${ }^{67}$

Die wichtigsten Unterschiede in den beiden Verfahren aus Sicht der Einbindung von Bürgern können wie folgt zusammengefasst werden: Der Autonomiekonvent sah eine vorgeschaltete Bürgerbeteiligungsphase (Open Space Veranstaltungen verteilt auf ganz Südtirol zur Frage „Welche Zukunft für unser Land?“) und thematische Workshops für Interessensgruppen vor. ${ }^{68}$ Die beiden Gremien des Autonomiekonvents beteiligten die Bürger auch direkt an den Arbeiten: 1) Das Forum der 100 setzte sich aus 100 Bürgern zusammen, die mittels eines geschichteten Losverfahrens - Geschlecht, Sprache, Alter - Südtirol im Kleinen darstellten. Aufgabe des Forums der 100 war es, die Ergebnisse der Open Space Veranstaltungen in acht Arbeitsgruppen zu vertiefen;69 2) Dem Konvent der 33, der sich zu 27 Arbeitssitzungen traf, gehörten neben Mandatsträgern, Experten und Interessensvertretern auch acht Entsandte aus dem Forum der 100 an. Im Trentino erarbeitete die Consulta, ein 25-köpfiges Gremium aus Experten, Mandatsträgern und Interessensvertretern, vorläufige Leitlinien zur Reform des Sonderstatuts und unterbreitete diese in einem sechsmonatigen Bürgerbeteiligungsprozess der Bevölkerung. Der zwischengeschaltete Bürgerbeteiligungsprozess in Form von Informations- bzw. Anhörungsveranstaltungen - im Vergleich zu den Bürgerbeteiligungsmomenten in Südtirol im Trentiner Landesgesetz Nr. 1/2016 nicht ausdrücklich vorgesehen, sondern von der Consulta selbst geregelt - erweckte nur geringes Interesse. ${ }^{70}$

67 Für eine detaillierte Analyse der Ergebnisse siehe Elisabeth Alber und Jens Woelk, ",Autonomie(reform) 2.0": parallele Verfahren partizipativer Demokratie zur Reform des Autonomiestatuts der Region Trentino-Südtirol«, in Europäisches Zentrum für Föderalismus-Forschung Tübingen (Hrsg.): Jahrbuch des Föderalismus 2018. Föderalismus, Subsidiarität und Regionen in Europa, (Nomos, 2018), $172 \mathrm{ff}$.

68 Knapp 2.000 Teilnehmer beteiligten sich an den neun Open Space, die zweisprachig (im Gadertal auch dreisprachig) abgehalten wurden. Insgesamt waren Italienischsprachige, Frauen und Jugendliche unterrepräsentiert. Für Details zu den Open Space Veranstaltungen und den thematischen Workshops mit Interessensgruppen siehe Elisabeth Alber, Marc Röggla und Vera Ohnewein, »,Autonomy Convention' and ,Consulta': Deliberative Democracy in Subnational Minority Contexts«, in European Centre for Minority Issues and Eurac Research (Hrsg.), European Yearbook of Minority Issues (Brill-Nijhoff, 2016), 194ff.

69 Das Forum der 100 traf sich über ein Jahr lang für sechs offizielle Treffen und mehrere informelle Arbeitsgruppentreffen.

70 Zwischen März und September 2017 nahmen 700 Personen teil, wobei nur 168 Personen von ihrem Rederecht Gebrauch machten. 
Was die Debatten in den Gremien angeht, so waren diese im Forum der 100 und dem Konvent der 33 politisch bedingt oft konfliktbeladen. Die konfliktorientierte Diskussionskultur, die im Widerspruch zu den konkordanzdemokratischen Grundlagen des Südtiroler Autonomiemodells steht, führte dazu, dass sich im Konvent der 33 einige Mitglieder mit ihren Minderheitenberichten (sämtlich von italienischsprachigen Mitgliedern verfasst) vom Abschlussdokument distanzierten. Zwar konnte eine Schlussabstimmung aufgund der Interpretation dieser Berichte als ausgesprochener Dissens zum allgemeinen Abschlussdokument vermieden werden, doch sind Minderheitenberichte und Konsensprinzip aus Sicht der deliberativen Demokratietheorie nicht vereinbar. Das Konsensprinzip war vom Landesgesetzgeber für den Autonomiekonvent als Arbeitsweise vorgeschrieben (Art. 1 Abs. 2 Landesgesetz Nr. 3/2015) - wie auch (!) die Möglichkeit der Verabschiedung von Minderheitenberichten. Das Verfassen der Minderheitenberichte kann als ein Übergang vom Konsensprinzip zur Logik von Mehrheitsentscheidungen gewertet werden und ist ein Indiz dafür, dass im Konvent der 33 das sprachgruppenübergreifende Autonomieverständnis nicht gegeben war. Auch im Forum der 100, welches parallel zum Konvent der 33 tagte und seine Ergebnisse am 12. Mai 2017 dem Konvent der 33 übermittelte, konnten nicht alle acht Arbeitsgruppen das Konsensprinzip einhalten. Die Arbeitsgruppe zum Schulsystem und den Ortsnamen konnte sich nicht auf ein gemeinsames Ergebnis einigen (mit einem Teil der Arbeitsgruppe, der die Einführung eines integrierten, mehrsprachigen Schultyps neben den Regelschultypen in deutscher und italienischer Sprache empfahl, während ein anderer Teil auf die strikte Trennung und dem Prinzip des Unterrichts in der Muttersprache beharrte). Da der Konsens jedoch in sieben von insgesamt acht Arbeitsgruppen erreicht werden konnte, lässt sich behaupten, dass der Dialog zwischen Sprachgruppen und Generationen im Forum der 100 funktioniert hat.

Die Consulta, weitgehend frei von landesgesetzlichen Vorgaben hinsichtlich ihrer Arbeitsweise, konnte konsensorientiert diskutieren. Im Trentino waren die Debatten aufgrund der geringeren Heterogenität innerhalb der Consulta (es gab keine Aufteilung nach Sprachgruppen, nur einen von der Trentiner Konferenz für Sprachminderheiten entsandten Vertreter, der ihre Interessen vertrat) und der anderen Arbeitsweise (akademische Referentenmethode zu Makrothemen mit anschließender Diskussion vs. - im Konvent der 33 - offener Diskussion ähnlich einer Parlamentsdebatte) weniger konfliktgeladen. Die Consulta konnte ihr Abschlussdokument trotz anfänglicher Minderheitenpositionen im vorläufigen Dokument der Leitlinien einstimmig dem Trentiner Landtag übergeben. Kritiker werfen der Consulta vor, dass diese eine zu stark am italienischen 
Regionalstaat angepasste und zu weit von den Südtiroler Vorstellungen der "Vollautonomie“ entfernten Vorstellung der Zukunft der Autonomie ausgearbeitet hat. ${ }^{71}$

Beide Gremien, der Konvent der 33 und die Consulta, entschieden sich für die Ausarbeitung eines Maßnahmen- und Empfehlungskatalogs und nicht für ein in Artikel gegliedertes endgültiges Dokument. ${ }^{72}$ Die Ergebnisse der Gremien des Autonomiekonvents, also auch der acht thematischen Arbeitsgruppen des Forums der 100, und der Consulta wurden im September 2017 (Südtirol) und Mai 2018 (Trentino) den jeweiligen Landtagen und Landeshauptmännern übergeben und bisher nicht weiter behandelt.

\section{Fazit}

Der Beitrag ist auf das Konzept der deliberativen Demokratie und die Institutionalisierung deliberativer Bürgerbeteiligungsverfahren in Italiens Regionen eingegangen und hat die Beispiele - Fallstudien organischer Normenwerke einerseits und Verfahren bei grundlegenden Reformen (in geteilten Gesellschaften) andererseits - kontextuell eingebettet in die Entwicklungen des italienischen Regionalismus und seiner federality.

Der Beitrag hat aufgezeigt, wie auch in italienischsprachigen Teildisziplinen der Sozialwissenschaften deliberativen Bürgerbeteiligungsverfahren mehrheitlich die theoriegeleitete Hoffnung zugeschrieben wird, politische Systeme in ihren Grundstrukturen und Entscheidungsfindungsprozessen durch die prozessuale Einbindung von Bürgern stabilisieren zu können. ${ }^{73}$

71 So insbesondere der Trentiner Verfassungsrechtler Roberto Toniatti, selbst Rechtsexperte im Konvent der 33 und dort neben der Mitarbeit am Mehrheitspapier Verfasser eines Minderheitenberichts (zur Präambel und dem Bezug auf die "christlichen Wurzeln“, und zum Selbstbestimmungsprinzip); zum Beispiel: Roberto Toniatti, »2018: Statuto. La storia snobbata«, Corriere del Trentino, 19. Juli 2018, 1.

72 In Südtirol verlangte das Landesgesetz zunächst ein endgültiges in Artikeln gegliedertes Dokument samt Begleitbericht (Art. 1 Abs. 2 c Landesgesetz Nr. 3/2015). Der Südtiroler Landtag hat am 22. Dezember 2016 eigens das Landesgesetz zum Autonomiekonvent geändert, um eine Artikelgliederung ausdrücklich auszuschließen (Art. 17 Abs. 1 Landesgesetz Nr. 27/2016).

73 Vgl. Umberto Allegretti, »Democrazia partecipativa: un contributo alla democratizzazione della democrazia«, in Umberto Allegretti (Hrsg.), Democrazia partecipativa - Esperienze e prospettive in Italia e in Europa (Firenze University Press, 2010), $5 \mathrm{ff}$. 
Vom input-orientierten Legitmitätsgewinn, den solche Verfahren der herkömmlichen auf dem Mehrheitsprinzip fußenden Entscheidungsfindung hinzusteuern, erhofft man sich, dass die Leistungsfähigkeit von Systemen durch eine responsive an den Interessen der Bürgerschaft orientierte Politikgestaltung der Mandatsträger gesteigert wird und das Vertrauen zwischen der Bevölkerung und den Institutionen wieder gefestigt wird. ${ }^{74}$

Lange war die institutionelle Ausgestaltung deliberativer Demokratie unterspezifiziert, wobei die Lehrmeinungen zur Institutionalisierung deliberativer Bürgerbeteiligungsverfahren auseinandergehen, in Italien und weltweit. Während die einen die „Konsultative“ als vierte Gewalt neben der Legislative, Exekutive und Judikative deuten, ${ }^{75}$ weisen die anderen (auch) auf die Risiken hin (beispielsweise die Kontrolle von oben durch die Letztentscheidungsinstanz oder Meinungspolarisierungen $)^{76}$. Sicherlich, aufgrund des Prinzips der freiwilligen (und meist ehrenamtlichen) Teilnahme, kommt es bei der Durchführung deliberativer Bürgerbeteiligungsverfahren zu einem Abbild der Realität, das von der Realität (stark) abweichen kann. Jedoch ist die Behauptung, dass ein Mehr an Beteiligungsrechten für politische Ungleichheit sorgt, genauso eine unzulässige Verallgemeinerung wie jene, dass deliberative Bürgerbeteiligungsverfahren ein Allheilmittel sind. Die Ungleichheit ist nämlich soziale Wirklichkeit und ursächlich keinem Beteiligungsverfahren zuzurechnen. ${ }^{77}$

Eines ist sicher: Dem Ideal der "guten Deliberation" kommt die Institutionalisierung deliberativer Bürgerbeteiligungsverfahren nur dann nahe, wenn die Kontextsensibilität für solche gegeben ist. Institutionelle Beharrungsmomente müssen bei der Ausgestaltung von Partizipationsverfahren überwunden werden. Herkömmliche Verfahren der Entscheidungsfindung, die die deliberativen Verfahren schlussendlich legitimieren und einsetzen, werden von denselben nämlich auch herausgefordert. Für den

74 Vgl. Francesco Palermo, »Participation, Federalism and Pluralism: Challenges to Decision Making and Responses by Constitutionalism «, in Cristina Fraenkel-Haeberle et al. (Hrsg.), Citizen Participation in Multi-Level Democracies (Brill-Nijhoff, 2015), $31 \mathrm{ff}$.

75 Vgl. Patrizia Nanz und Claus Leggewie, Die Konsultative (Verlag Klaus Wagenbach, 2016).

76 Vgl. Luigi Bobbio, »Dilemmi della democrazia partecipativa«, (2006) 4 Democrazia e Diritto 11ff. Cass Sunstein, »The Law of Group Polarization«, (2002) 10(2) Journal of Political Philosophy $175 \mathrm{ff}$.

77 Angelika Vetter und Uwe Remer-Bollow, Bürger und Beteiligung in der Demokratie (VS Springer, 2017), 296. 
„Erfolg ${ }^{678}$ deliberativer Bürgerbeteiligungsverfahren sind außerdem noch folgende zwei Aspekte wichtig: Erstens, inwieweit die Teilnehmer nutzenmaximierend und gemeinwohlorientiert agieren. Zweitens, inwieweit die Letztentscheidungsinstanz den Ergebnissen Rechnung trägt bzw. Abweichungen davon begründet. Die Beantwortung dieser Fragen bleibt derzeit (für Italien) noch weitgehend ein wissenschaftliches Desiderat.

78 Es gibt keine Einigkeit zur Frage, ab wann man von einem gelungenen vollständigen deliberativen Prozess sprechen kann. Parkinson vertritt zum Beispiel die höchst anspruchsvolle These, dass ein deliberativer Prozess nur dann vollständig ist, sobald er alle Stufen des Entscheidungsprozesses durchlaufen hat. John Parkinson, »Democratizing deliberative systems", in John Parkinson und Jane Mansbridge (Hrsg.), Deliberative systems: Deliberative democracy at the large scale (Cambridge University Press, 2012). 